\title{
Design of Delay Observer-based Controllers for Uncertain Time-lag Systems
}

\author{
MAGDI S. MAHMOUD* and MOHAMED ZRIBI \\ Department of Electrical and Computer Engineering, Kuwait University, \\ P. O. Box 5969, Safat-13060, Kuwait
}

(Received 15 October 1998; In final form 24 February 1999)

\begin{abstract}
In this paper, the problem of designing observers and observer-based controllers for a class of uncertain systems with input and state time lags is considered. We construct delay-type observers in which both the instantaneous as well as the delayed measurements are utilized. Using feedback control based on the reconstructed state, the behavior of the closed-loop system is investigated. It is established that the uncertain time-lag system with delay observer-based control is asymptotically stable. Expressions for the gain matrices are given based on two linear-matrix inequalities. A numerical example is given to illustrate the theoretical developments.
\end{abstract}

Keywords: Design; Observer; Controller; Time-delay system; LMI

\section{INTRODUCTION}

The problem of state reconstruction for linear dynamic systems has received considerable attention for more than three decades. For deterministic systems with known dynamic models, the celebrated results of Leunberger [1] have provided the fundamental thrust upon which the theory of state observers is by now well developed [2]. When the dynamic models have uncertain parameters, the available results are limited to some cases including matched uncertainties [3], dyadic-type uncertainties [4] and norm-bounded uncertainties [5].

\footnotetext{
* Corresponding author. Tel.: 965 481-1188, ext. 5835. Fax: 965-481-7451.

E-mail: magdi(a)eng.kuniv.edu.kw.
} 
For the case of uncertain dynamical systems with state and/or input delays, it seems that the results are very scattered. Nonlinear and insensitive observers have been developed in [6] for linear systems with mismatched uncertainties and state delays. $H_{\infty}$-controllers using fullorder observers have been studied in [7] when the state and the input delay factors are known. Modal-type observers for time-lag systems have been developed in [8] and [9]. The present work builds on the forgoing results and extends them. It deals with the problem of designing observers and observer-based controllers for a class of nominally linear systems with state and input lags. We construct a delay-type observer in which both instantaneous and delayed information is used to reconstruct the state of the system. We then establish that, the uncertain time-lag system with delay observer-based control is asymptotically stable. Expressions for the gain matrices are given based on two linearmatrix inequalities (LMIs). A numerical example is given to illustrate the theoretical developments.

\section{Notations and Facts}

In the sequel, we denote by $W^{\mathrm{t}}$ and $W^{-1}$ the transpose and the inverse of any square matrix $W$. We use $W>0(W<0)$ to denote a positive(negative-) definite matrix $W$. We let $I$ be a unit matrix of appropriate dimensions. Sometimes, the arguments of a function will be omitted in the analysis when no confusion can arise.

\section{Fact 1 (Schur Complement)}

Given the constant matrices $\Omega_{1}, \Omega_{2}, \Omega_{3}$ where $\Omega_{1}=\Omega_{1}^{\mathrm{t}}$ and $0<\Omega_{2}=\Omega_{2}^{\mathrm{t}}$ then $\Omega_{1}+\Omega_{3}^{\mathrm{t}} \Omega_{2}^{-1} \Omega_{3}<0$ if and only if

$$
\left[\begin{array}{cc}
\Omega_{1} & \Omega_{3}^{\mathrm{t}} \\
\Omega_{3} & -\Omega_{2}
\end{array}\right]<0 \text { or }\left[\begin{array}{cc}
-\Omega_{2} & \Omega_{3} \\
\Omega_{3}^{\mathrm{t}} & \Omega_{1}
\end{array}\right]<0
$$

\section{Fact 2}

For any real matrices $\Sigma_{1}$ and $\Sigma_{2}$ with appropriate dimensions, it follows that

$$
\Sigma_{1}^{\mathrm{t}} \Sigma_{2}+\Sigma_{2}^{\mathrm{t}} \Sigma_{1} \leq \alpha \Sigma_{1}^{\mathrm{t}} \Sigma_{1}+\alpha^{-1} \Sigma_{2}^{\mathrm{t}} \Sigma_{2}, \quad \alpha>0 .
$$




\section{Fact 3}

Let $\Sigma_{1}, \Sigma_{2}$ and $\Sigma_{3}$ be real constant matrices of compatible dimensions, and $H(t)$ be a real matrix function satisfying $H^{\mathrm{t}}(t) H(t) \leq \psi I ; \psi>0$. Then for any $\epsilon>0$, the following inequality holds,

$$
\Sigma_{1} \Sigma_{2} H(t) \Sigma_{3}+\Sigma_{3}^{\mathrm{t}} H^{\mathrm{t}}(t) \Sigma_{2}^{\mathrm{t}} \Sigma_{1}^{\mathrm{t}} \leq \epsilon^{-1} \Sigma_{1} \Sigma_{2} \Sigma_{2}^{\mathrm{t}} \Sigma_{1}^{\mathrm{t}}+\epsilon \psi \Sigma_{3}^{\mathrm{t}} \Sigma_{3} .
$$

\section{PROBLEM DESCRIPTION}

In this section, we consider a class of uncertain systems with state and input lags represented by a state space model of the form:

$$
\begin{aligned}
\dot{x}(t)= & {\left[A_{\mathrm{o}}+\Delta A(t)\right] x(t)+\left[B_{\mathrm{o}}+\Delta B(t)\right] u(t) } \\
& +\left[D_{\mathrm{o}}+\Delta D(t)\right] x(t-\tau)+E_{\mathrm{o}} u(t-\eta), \\
y(t)= & {\left[C_{\mathrm{o}}+\Delta C(t)\right] x(t), } \\
x(t)= & \psi_{1}(t) \quad \forall t \in[-\max (\tau, \eta), 0],
\end{aligned}
$$

where $t \in \Re$ is the time, $x(t) \in \Re^{n}$ is the state, $u(t) \in \Re^{m}$ is the control input; $y(t) \in \Re^{p}$ is the measured output, and $\tau, \eta$ are known constant scalars representing the amount of delays in the state and at the input of the system, respectively. The matrices $A_{\mathrm{o}} \in \Re^{n \times n}, B_{\mathrm{o}} \in \Re^{n \times m}$, $D_{\mathrm{o}} \in \Re^{n \times n}, \quad E_{\mathrm{o}} \in \Re^{n \times m}$ and $C_{\mathrm{o}} \in \Re^{p \times n}$ are real constant matrices representing the nominal plant with the pair $\left(A_{\mathrm{o}}, B_{\mathrm{o}}\right)$ being controllable, and the pair $\left(A_{\mathrm{o}}, C_{\mathrm{o}}\right)$ being observable. The uncertain matrices $\Delta A(t), \Delta B(t), \Delta C(t)$ and $\Delta D(t)$ are assumed to be represented by

$$
\begin{aligned}
{[\Delta A(t) \Delta B(t)] } & =H_{1} F(t)\left[G_{1} \quad G_{2}\right] ; \quad \Delta C(t)=H_{2} F(t) G_{1} ; \\
\Delta D(t) & =H_{3} F(t) G_{3} ; \quad F^{\mathrm{t}}(t) F(t) \leq I .
\end{aligned}
$$

The initial functions of system (1)-(2) are specified as $x_{\mathrm{o}} \in C([-\tau, 0]$; $\left.\Re^{\prime \prime}\right)$ and $u_{\mathrm{o}} \in C\left([-\eta, 0] ; \Re^{\prime \prime \prime}\right)$.

The problem of interest is to design observer-based controllers for the time-lag uncertain-system (1)-(2) with norm-bounded uncertainties satisfying (3) and (4). 
Before proceeding further, the following definitions are given.

DeFINITION 1 The uncertain time-delay system (1)-(2) is said to be robustly stable if the null solution $x(t) \equiv 0$ of $(1)$ with $u(t) \equiv 0$ is globally uniformly asymptotically stable for all admissible uncertainties satisfying (3) and (4).

DEFINITION 2 The uncertain time-delay system (1)-(2) is said to be robustly stabilizable if there exists a feedback controller $u(t)=K[x(t)]$, such that the resulting closed-loop system is robustly stable in the sense of Definition 1.

The design objectives are:

(O1) to reconstruct the state $x(t)$ based on the input and/or output measurements,

(O2) to determine the state-feedback gain matrix such that the closedloop controlled system is asymptotically stable,

(O3) to provide an efficient procedure to compute the controller and the observer gains.

These objectives should be fulfilled for all admissible uncertainties satisfying (3) and (4).

There are two generic groups of observers that can be used to fulfill objective (O1). Hence, observers can be classified as delay-less observers or delay observers.

1. Delay-less Observers These observers are observers that depend only on the instantaneous input-output measurements. Examples of such observers can be found in [8-10]. Modal-type observers have been developed by Levy-Ramos and Pearson [8], and Trinh and Aldeen [9] for time-lag systems. Jabbari and Schmitendorf [3] have developed a full state observer for uncertain systems. A delay-less Leunberger-observer for uncertain systems with input and state delays has been developed by Mahmoud and Zribi [10].

2. Delay Observers These observers are such that the observer state depends on both the instantaneous and the delayed input-output measurements. 
Conceptually, the two groups of observers are different at least from the amount of input information for which the second group demands more.

In this paper will concentrate on delay observers for the class of time-lag system (1)-(2). The rationale behind the construction of these observers is the desire to utilize all the available input-output instantaneous and delayed information in estimating the state variables. In fact, the use of delayed state in feedback control design has been shown to provide satisfactory performance [11].

By utilizing all the information at hand (both instantaneous and delayed) we come up with the generalized full-order linear observer:

$$
\dot{\hat{x}}(t)=A_{\mathrm{o}} \hat{x}(t)+B_{\mathrm{o}} u(t)+D_{\mathrm{o}} \hat{x}(t-\tau)+E_{\mathrm{o}} u(t-\eta)+L\left[y(t)-C_{\mathrm{o}} \hat{x}(t)\right],
$$

where $L \in \Re^{n \times p}$ is the observer gain matrix to be determined later.

Remark 1 There are two possible special cases of observer (5). The first case is given by:

$$
\dot{\hat{x}}(t)=A_{\mathrm{o}} \hat{x}(t)+B_{\mathrm{o}} u(t)+D_{\mathrm{o}} \hat{x}(t-\tau)+L\left[y(t)-C_{\mathrm{o}} \hat{x}(t)\right],
$$

where $L \in \Re^{n \times p}$ is the observer gain matrix. This observer uses the delayed state measurements. The second observer is such that:

$$
\dot{\hat{x}}(t)=A_{\mathrm{o}} \hat{x}(t)+B_{\mathrm{o}} u(t)+E_{\mathrm{o}} u(t-\eta)+L\left[y(t)-C_{\mathrm{o}} \hat{x}(t)\right]
$$

where $L \in \Re^{n \times p}$ is the observer gain matrix. Note that this observer uses the delayed input measurements.

The end result of observer (5) is to develop $\hat{x}(t)$ as a good replica of $x(t)$. Hence it can be used for state-feedback design. A state-feedback controller of the form

$$
u(t)=K \hat{x}(t)
$$

where $K \in \Re^{m \times n}$ is the feedback gain matrix will be designed such that the closed-loop controlled system is asymptotically stable. 


\section{STABILITY OF THE CLOSED-LOOP SYSTEM}

We start by defining the state error $e(t)$ such that:

$$
e(t)=x(t)-\hat{x}(t)
$$

The error dynamics depends on the type of observer being used. An augmented system can be formed in the $(x, e)$ space in terms of the composite state vector

$$
x_{\mathrm{t}}(t)=\left[\begin{array}{l}
x(t) \\
e(t)
\end{array}\right] \in \Re^{2 n}
$$

Note that (8)-(10) imply that

$$
u(t)=K_{t} x_{t}(t)
$$

where $K_{t}=[K-K] \in \Re^{m \times 2 n}$. By differentiating (9), making use of system (1)-(2) in addition to the observer dynamics (5) and manipulating, we can cast the augmented system into the general form:

$$
\begin{aligned}
\dot{x}_{t}(t)= & {\left[A_{t}+\Delta A_{t}(t)\right] x_{t}(t)+\left[B_{t}+\Delta B_{t}(t)\right] u(t) } \\
& +\left[D_{t}+\Delta D_{t}(t)\right] x_{t}(t-\tau)+E_{t} u(t-\eta),
\end{aligned}
$$

where

$$
\begin{aligned}
A_{t} & =\left[\begin{array}{cc}
A_{\mathrm{o}} & 0 \\
0 & A_{\mathrm{o}}-L C_{\mathrm{o}}
\end{array}\right], \quad B_{t}=\left[\begin{array}{c}
B_{\mathrm{o}} \\
0
\end{array}\right], \\
D_{t} & =\left[\begin{array}{cc}
D_{\mathrm{o}} & 0 \\
0 & D_{\mathrm{o}}
\end{array}\right], \quad E_{t}=\left[\begin{array}{c}
E_{\mathrm{o}} \\
0
\end{array}\right], \\
\Delta A_{t} & =\left[\begin{array}{cc}
\Delta A(t) & 0 \\
\Delta A(t)-L \Delta C(t) & 0
\end{array}\right]=\left[\begin{array}{c}
H_{1} \\
H_{1}-L H_{2}
\end{array}\right] F(t)\left[\begin{array}{ll}
G_{1} & 0
\end{array}\right], \\
\Delta B_{t} & =\left[\begin{array}{l}
\Delta B(t) \\
\Delta B(t)
\end{array}\right]=\left[\begin{array}{l}
H_{1} \\
H_{1}
\end{array}\right] F(t) G_{2}, \\
\Delta D_{t} & =\left[\begin{array}{ll}
\Delta D(t) & 0 \\
\Delta D(t) & 0
\end{array}\right]=\left[\begin{array}{l}
H_{3} \\
H_{3}
\end{array}\right] F(t)\left[\begin{array}{ll}
G_{3} & 0
\end{array}\right] .
\end{aligned}
$$


In order to investigate the stability behavior of the uncertain timelag system (12), we use the Lyapunov-Krasovskii functional approach [12] and introduce the following storage functional:

$$
\begin{aligned}
V\left(x_{t}\right)= & x_{t}^{\mathrm{t}}(t) W_{1} x_{t}(t)+\int_{-\tau}^{0} x_{t}^{\mathrm{t}}(t+\alpha) W_{2} x_{t}(t+\alpha) \mathrm{d} \alpha \\
& +\int_{-\eta}^{0} x_{t}^{\mathrm{t}}(t+\beta) W_{3} x_{t}(t+\beta) \mathrm{d} \beta,
\end{aligned}
$$

where the weighting matrices are such that $0<W_{1}=W_{1}^{\mathrm{t}} \in \Re^{2 n \times 2 n}$, $0<W_{2}=W_{2}^{\mathrm{t}} \in \Re^{2 n \times 2 n}$ and $0<W_{3}=W_{3}^{\mathrm{t}} \in \Re^{2 n \times 2 n}$. Note that, $V\left(x_{t}\right)>0$ for all $x_{t}(t) \neq 0$ and $V\left(x_{t}\right)=0$ for $x_{t}(t)=0$. Let

$$
W_{1}=\left[\begin{array}{cc}
W_{11} & 0 \\
0 & W_{12}
\end{array}\right], \quad W_{2}=\left[\begin{array}{cc}
W_{21} & 0 \\
0 & W_{22}
\end{array}\right], \quad W_{3}=\left[\begin{array}{cc}
W_{31} & 0 \\
0 & W_{32}
\end{array}\right] \text {. }
$$

The following theorem summarizes the main result.

THEOREM 1 Consider the system (1)-(2) satisfying (3) and (4) and the linear observer (5). Then, given $\tau$ and $\eta$, the closed-loop controlled system (12) is robustly stabilizable if there exist matrices $0<P=P^{\mathrm{t}}$, $0<Q=Q^{\mathrm{t}}, 0<S_{j}=S_{j}^{\mathrm{t}}(j=1, \ldots, 5)$ and scalars $\alpha_{1}>0, \alpha_{2}>0, \alpha_{3}>0$ satisfying the following LMIs:

$$
\left.\begin{array}{cccccc}
A_{\mathrm{o}} P+P A_{\mathrm{o}}^{\mathrm{t}}+\Pi_{1} & \vdots & P & \sqrt{3} B_{\mathrm{o}} & E_{\mathrm{o}} & D_{\mathrm{o}} \\
\cdots & \vdots & -S_{5}^{-1} & 0 & 0 & 0 \\
P & \vdots & 0 & -S_{1} & 0 & 0 \\
\sqrt{3} B_{\mathrm{o}}^{\mathrm{t}} & \vdots & 0 & 0 & -S_{4}^{-1} & 0 \\
E_{\mathrm{o}}^{\mathrm{t}} & \vdots & 0 & 0 & 0 & -S_{2}
\end{array}\right]<0,
$$


and

$$
\left[\begin{array}{ccccc}
\widehat{A} Q+Q \widehat{A}^{\mathrm{t}}+\Phi+Q R_{2} Q & \vdots & Q K^{\mathrm{t}} & Q & D_{\mathrm{o}} \\
\cdots & \vdots & -S_{1}^{-1} & 0 & 0 \\
K Q & \vdots & 0 & -R_{1}^{-1} & 0 \\
Q & \vdots & 0 & 0 & -S_{3}
\end{array}\right]<0,
$$

where

$$
\begin{aligned}
\Pi_{1} & =\alpha_{1}^{-1} H_{1} H_{1}^{\mathrm{t}}+\alpha_{2}^{-1} H_{1} H_{1}^{\mathrm{t}}+\alpha_{3}^{-1} H_{3} H_{3}^{\mathrm{t}}, \\
\varphi & =H_{12}^{\mathrm{t}}\left(H_{12} H_{12}^{\mathrm{t}}\right)^{-1} \quad \text { with } H_{12}=\alpha_{1}^{-1} H_{2} H_{1}^{\mathrm{t}}, \\
\widehat{A} & =A_{\mathrm{o}}-\Pi_{1} \varphi C_{\mathrm{o}}-2 \Pi_{1} \varphi \theta \varphi^{\mathrm{t}} B_{\mathrm{o}} K+2 B_{\mathrm{o}} K, \\
R_{2} & =2 K^{\mathrm{t}} B_{\mathrm{o}}^{\mathrm{t}} \varphi C_{\mathrm{o}}+2 C_{\mathrm{o}}^{\mathrm{t}} \varphi^{\mathrm{t}} B_{\mathrm{o}} K+4 K^{\mathrm{t}} B_{\mathrm{o}}^{\mathrm{t}} \varphi \theta \varphi^{\mathrm{t}} B_{\mathrm{o}} K, \\
\Phi & =-\Pi_{1}+\Pi_{1} \varphi \theta \varphi^{\mathrm{t}} \Pi_{1}, \quad \theta=\alpha_{1}^{-1} H_{2} H_{2}^{\mathrm{t}} .
\end{aligned}
$$

Moreover, the observer and controller gain matrices are given by

$$
\begin{aligned}
& K=S_{1}^{-1} B_{\mathrm{o}}^{\mathrm{t}} P^{-1}, \\
& L=\left(-2 Q K^{\mathrm{t}} B_{\mathrm{o}}^{\mathrm{t}}+\Pi_{1}\right) \varphi .
\end{aligned}
$$

Proof By differentiating (18) along the solutions of (12) using (13)(17), the Lyapunov derivative $\dot{V}\left(x_{t}\right)$ can be expressed as:

$$
\dot{V}\left(x_{t}\right)<Z^{\mathrm{t}}(t) \Omega_{t} Z(t)
$$

where the extended state vector $Z(t)=\left[x_{t}^{\mathrm{t}}(t) x_{t}^{\mathrm{t}}(t-\tau) x_{t}^{\mathrm{t}}(t-\eta)\right]^{\mathrm{t}}$, and

$$
\begin{aligned}
& \Omega_{t}=
\end{aligned}
$$

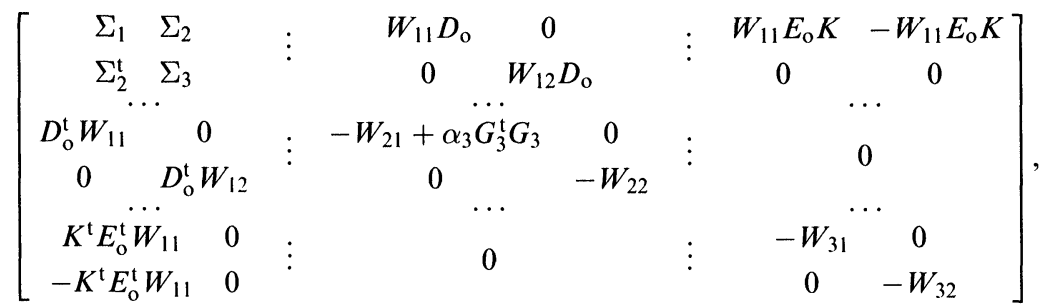


with

$$
\begin{aligned}
\Sigma_{1}= & W_{11} A_{\mathrm{o}}+A_{\mathrm{o}}^{\mathrm{t}} W_{11}+K^{\mathrm{t}} B_{\mathrm{o}}^{\mathrm{t}} W_{11}+W_{11} B_{\mathrm{o}} K+W_{21}+W_{31} \\
& +\alpha_{1} G_{1}^{\mathrm{t}} G_{1}+\alpha_{2} K^{\mathrm{t}} G_{2}^{\mathrm{t}} G_{2} K+W_{11} \Pi_{1} W_{11}, \\
\Sigma_{2}= & -W_{11} B_{\mathrm{o}} K+W_{11}\left[\alpha_{1}^{-1} H_{1}\left(H_{1}^{\mathrm{t}}-H_{2}^{\mathrm{t}} L^{\mathrm{t}}\right)+\alpha_{2}^{-1} H_{1} H_{1}^{\mathrm{t}}\right. \\
& \left.+\alpha_{3}^{-1} H_{3} H_{3}^{\mathrm{t}}\right] W_{12}-\alpha_{2} K^{\mathrm{t}} G_{2}^{\mathrm{t}} G_{2} K, \\
\Sigma_{3}= & W_{12}\left(A_{\mathrm{o}}-L C_{\mathrm{o}}\right)+\left(A_{\mathrm{o}}-L C_{\mathrm{o}}\right)^{\mathrm{t}} W_{12}+W_{22}+W_{32} \\
& +\alpha_{2} K^{\mathrm{t}} G_{2}^{\mathrm{t}} G_{2} K+W_{12} \Pi_{3} W_{12}, \\
\Pi_{3}= & \alpha_{1}^{-1}\left(H_{1}-L H_{2}\right)\left(H_{1}^{\mathrm{t}}-H_{2}^{\mathrm{t}} L^{\mathrm{t}}\right)+\alpha_{2}^{-1} H_{1} H_{1}^{\mathrm{t}}+\alpha_{3}^{-1} H_{3} H_{3}^{\mathrm{t}} .
\end{aligned}
$$

Expansion of $\Omega_{t}$ leads to

$$
\left[\begin{array}{ll}
\Xi_{11} & \Xi_{21} \\
\Xi_{21}^{\mathrm{t}} & \Xi_{31}
\end{array}\right]<0,
$$

where

$$
\begin{aligned}
& \Xi_{11}=\Sigma_{1}+W_{11} \Pi_{2} W_{11}, \\
& \Xi_{21}=\Sigma_{2}, \\
& \Xi_{31}=\Sigma_{3}+W_{12} D_{\mathrm{o}} W_{22}^{-1} D_{\mathrm{o}}^{\mathrm{t}} W_{12}, \\
& \Pi_{2}=E_{\mathrm{o}}\left(K W_{31}^{-1} K^{\mathrm{t}}\right) E_{\mathrm{o}}^{\mathrm{t}}+E_{\mathrm{o}}\left(K W_{32}^{-1} K^{\mathrm{t}}\right) E_{\mathrm{o}}^{\mathrm{t}}+D_{\mathrm{o}} S_{2}^{-1} D_{\mathrm{o}}^{\mathrm{t}}
\end{aligned}
$$

A sufficient condition to satisfy (35) is that

$$
\Xi_{11}<0, \quad \Xi_{31}<0, \quad \Xi_{21}=0 \text {. }
$$

Choosing $K$ as given by (27), and letting $S_{1}=\alpha_{2} G_{2}^{\mathrm{t}} G_{2}$ and $S_{5}=W_{21}+$ $W_{31}+\alpha_{1} G_{1}^{\mathrm{t}} G_{1}$, the inequality $\Xi_{11}<0$ reduces to

$$
W_{11} A_{\mathrm{o}}+A_{\mathrm{o}}^{\mathrm{t}} W_{11}+3 W_{11} B_{\mathrm{o}} S_{1}^{-1} B_{\mathrm{o}}^{\mathrm{t}} W_{11}+S_{5}+W_{11}\left(\Pi_{1}+\Pi_{2}\right) W_{11}<0
$$

Pre-multiplying and post-multiplying the above inequality by $W_{11}^{-1}$, and letting $P=W_{11}^{-1}, S_{2}=W_{21}-\alpha_{3} G_{3}^{\mathrm{t}} G_{3}, S_{4}=K\left(W_{31}^{-1}+W_{32}^{-1}\right) K^{\mathrm{t}}$, we obtain

$$
A_{\mathrm{o}} P+P A_{\mathrm{o}}^{\mathrm{t}}+\Pi_{1}+P S_{5} P+3 B_{\mathrm{o}} S_{1}^{-1} B_{\mathrm{o}}^{\mathrm{t}}+E_{\mathrm{o}} S_{4} E_{\mathrm{o}}^{\mathrm{t}}+D_{\mathrm{o}} S_{2}^{-1} D_{\mathrm{o}}^{\mathrm{t}}<0
$$


which when arranged using the Schur complement formula yields block form (20a).

From (37), using (32) and setting $\Xi_{21}=0$, yields

$$
-2 W_{11} B_{\mathrm{o}} K+W_{11} \Pi_{1} W_{12}-\alpha_{1}^{-1} W_{11} H_{1} H_{2}^{\mathrm{t}} L^{\mathrm{t}} W_{12}=0 .
$$

By letting $Q=W_{12}^{-1}$, and pre-multiplying the above inequality by $W_{11}^{-1}$, and post-multiplying the result by $W_{12}^{-1}$, one obtains,

$$
-2 B_{0} K Q+\Pi_{1}-\alpha_{1}^{-1} H_{1} H_{2}^{\mathrm{t}} L^{\mathrm{t}}=0
$$

or equivalently stated,

$$
L H_{12}=-2 Q K^{\mathrm{t}} B_{\mathrm{o}}^{\mathrm{t}}+\Pi_{1} .
$$

Assuming that $H_{12} H_{12}^{\mathrm{t}}$ is nonsingular, and letting $\varphi=H_{12}^{\mathrm{t}}\left(H_{12} H_{12}^{\mathrm{t}}\right)^{-1}$, one directly obtains $L$ as given by (28).

Finally considering the inequality $\Xi_{31}<0$. With $R_{1}=W_{32}+W_{22}$, starting from (38) and using (33), (22)-(26), we get

$$
\begin{aligned}
& W_{12} \widehat{A}+\widehat{A}^{\mathrm{t}} W_{12}+W_{12} \Phi W_{12}+R_{1}+R_{2}+K^{\mathrm{t}} S_{1} K \\
& \quad+W_{12} D_{\mathrm{o}} W_{22}^{-1} D_{\mathrm{o}}^{\mathrm{t}} W_{12}<0 .
\end{aligned}
$$

Pre-multiplying and post-multiplying the above inequality by $W_{12}^{-1}$, one obtains,

$$
\widehat{A} Q+Q \widehat{A}^{\mathrm{t}}+\Phi+Q R_{1} Q+Q R_{2} Q+Q K^{\mathrm{t}} S_{1} K Q+D_{\mathrm{o}} S_{3}^{-1} D_{\mathrm{o}}^{\mathrm{t}}<0 .
$$

Inequality (47) can be easily put in the block form (21) by using the Schur Complement formula.

Remark 2 To implement Theorem 1, we apply the following procedure:

(1) Read in the data $A_{\mathrm{o}}, B_{\mathrm{o}}, \ldots, H_{1}, \ldots$

(2) Solve inequality (20) for $P$, then compute the gain matrix $K$ from (27). 
(3) Solve inequality (21) for $Q$, then compute the gain matrix $L$ from (28).

(4) Simulate the performance of system (12) when controller (11) is used.

COROLlaRY 1 Consider the uncertain time-lag system (1)-(2) with observer (6) and controller (11). The resulting observer-based controlled system is asymptotically stable with controller gain matrix $K$ as given by (27) and observer gain matrix L such that:

$$
L=\left(-2 Q K^{\mathrm{t}} B_{\mathrm{o}}^{\mathrm{t}}+\Pi_{1}+E_{\mathrm{o}} S_{4} E_{\mathrm{o}}^{\mathrm{t}}\right) \varphi
$$

where the matrix $0<Q=Q^{\mathrm{t}}$ satisfies:

$$
\left[\begin{array}{cccccc}
\widehat{A} Q+Q \widehat{A}^{\mathrm{t}}+\Phi+Q R_{2} Q & \vdots & Q K^{\mathrm{t}} & Q & D_{\mathrm{o}} & E_{\mathrm{o}} \\
\cdots & \vdots & -S_{1}^{-1} & 0 & 0 & 0 \\
K Q & \vdots & 0 & -R_{1}^{-1} & 0 & 0 \\
Q & \vdots & 0 & 0 & -S_{3} & 0 \\
D_{\mathrm{o}}^{\mathrm{t}} & \vdots & 0 & 0 & 0 & -S_{4}^{-1}
\end{array}\right]<0 .
$$

Proof The proof follows parallel lines to the proof of Theorem 1 with

$$
\begin{aligned}
& \Xi_{21}=\Sigma_{2}+W_{11} E_{\mathrm{o}} S_{4} E_{\mathrm{o}}^{\mathrm{t}} W_{12}, \\
& \Xi_{31}=\Sigma_{3}+W_{12} E_{\mathrm{o}} S_{4} E_{\mathrm{o}}^{\mathrm{t}} W_{12}+W_{12} D_{\mathrm{o}} W_{22}^{-1} D_{\mathrm{o}}^{\mathrm{t}} W_{12},
\end{aligned}
$$

and all the other quantities remain unchanged.

COROLlary 2 Consider the uncertain time-lag system (1)-(2) with observer (7) and controller (11). The resulting observer-based controlled system is asymptotically stable with controller gain matrix Kas given by (27) and observer gain matrix L expressed as:

$$
L=\left(-2 Q K^{\mathrm{t}} B_{\mathrm{o}}^{\mathrm{t}}+\Pi_{1}+D_{\mathrm{o}} S_{2}^{-1} D_{\mathrm{o}}^{\mathrm{t}}\right) \varphi
$$


where the matrix $0<Q=Q^{\mathrm{t}}$ satisfies:

$$
\left[\begin{array}{ccccc}
\widehat{A} Q+Q \widehat{A}^{\mathrm{t}}+\Phi+Q R_{2} Q & \vdots & Q K^{\mathrm{t}} & Q & D_{\mathrm{o}} \\
\cdots & \vdots & \cdots & \cdots & \cdots \\
K Q & \vdots & -S_{1}^{-1} & 0 & 0 \\
Q & \vdots & 0 & -R_{1}^{-1} & 0 \\
D_{\mathrm{o}}^{\mathrm{t}} & \vdots & 0 & 0 & -S_{2}
\end{array}\right]<0 .
$$

Proof The proof follows parallel lines to the proof of Theorem 1 using

$$
\begin{aligned}
& \Xi_{21}=\Sigma_{2}+W_{11} D_{\mathrm{o}} S_{2}^{-1} D_{\mathrm{o}}^{\mathrm{t}} W_{12}, \\
& \Xi_{31}=\Sigma_{3}+W_{12} D_{\mathrm{o}} S_{2}^{-1} D_{\mathrm{o}}^{\mathrm{t}} W_{12},
\end{aligned}
$$

and all the other quantities remain unchanged.

Remark 3 In view of Theorem 1, the results of Corollaries 1 and 2 are expected due to two reasons:

(1) The controller (11) remains the same, hence $K$ must be the same in all cases.

(2) The change takes place in the expression of $L$ as result of changing the observer structure. More importantly, it is noted that the more information used to process the observer dynamics, the lesser will be the effort to determine the observer gain.

\section{SIMULATION EXAMPLE}

Consider the following third-order system:

$$
\begin{aligned}
\dot{x}(t)= & {\left[A_{\mathrm{o}}+\Delta A(t)\right] x(t)+\left[B_{\mathrm{o}}+\Delta B(t)\right] u(t) } \\
& +\left[D_{\mathrm{o}}+\Delta D(t)\right] x(t-\tau)+E_{\mathrm{o}} u(t-\eta), \\
y(t)= & {\left[C_{\mathrm{o}}+\Delta C(t)\right] x(t), }
\end{aligned}
$$


with

$$
\begin{aligned}
& {\left[\begin{array}{ll}
\Delta A(t) & \Delta B(t)
\end{array}\right]=H_{1} F(t)\left[\begin{array}{ll}
G_{1} & G_{2}
\end{array}\right], \quad \Delta C(t)=H_{2} F(t) G_{1},} \\
& \Delta D(t)=H_{3} F(t) G_{3},
\end{aligned}
$$

where

$$
\begin{aligned}
& A_{\mathrm{o}}=\left[\begin{array}{ccc}
-3 & 2 & 0 \\
1 & -2 & -1 \\
3 & 0 & -6
\end{array}\right] ; \quad B_{\mathrm{o}}=\left[\begin{array}{cc}
1 & 1 \\
4 & 3 \\
2 & -1
\end{array}\right] ; \quad C_{\mathrm{o}}=\left[\begin{array}{lll}
1 & 0 & 0
\end{array}\right] ; \\
& D_{\mathrm{o}}=\left[\begin{array}{ccc}
-1 & 0 & 1 \\
1 & 1 & 0 \\
0 & 0 & 2
\end{array}\right] ; \quad E_{\mathrm{o}}=\left[\begin{array}{cc}
0.1 & 0.5 \\
0.2 & 0.7 \\
0.6 & 0.2
\end{array}\right] \\
& H_{1}=\left[\begin{array}{cc}
0.2 & 0.4 \\
0.3 & -0.4 \\
0.1 & 0.2
\end{array}\right] ; \quad H_{2}=\left[\begin{array}{ll}
0.1 & -0.3
\end{array}\right] ; \quad H_{3}=\left[\begin{array}{cc}
-1.0 & 0.5 \\
0.3 & 0.5 \\
0 & 1.0
\end{array}\right] \text {; } \\
& G_{1}=\left[\begin{array}{ccc}
1.0 & 0.5 & -1.0 \\
0.8 & -1.0 & 0.2
\end{array}\right] ; \quad G_{2}=\left[\begin{array}{cc}
-1.0 & -0.5 \\
-1.0 & 0.7
\end{array}\right] ; \\
& G_{3}=\left[\begin{array}{ccc}
0.4 & 0.4 & 0.6 \\
-0.5 & 0.6 & -0.7
\end{array}\right] ; \quad F(t)=\left[\begin{array}{cc}
0.5 \sin (2 t) & 0.3 \sin (t) \\
-0.4 \sin (t) & 0.6 \sin (3 t)
\end{array}\right] \text {. }
\end{aligned}
$$

The amount of delays in the state and at the input of the system are such that $\tau=0.4$ and $\eta=0.2$.

It is desired to design an observer-based controller for this uncertain system. The results of Theorem 1 are used to design the observer-based controller.

Selecting the scalars $\alpha_{1}, \alpha_{2}$ and $\alpha_{3}$ such that $\alpha_{1}=0.0001, \alpha_{2}=10$ and $\alpha_{3}=1$, and then solving the matrix inequality (20) by using the LMI-Control Toolbox [13] gives:

$$
P=\left[\begin{array}{ccc}
1729.9 & 605.2 & 629.4 \\
605.2 & 1951.2 & 168.0 \\
629.4 & 168.0 & 981.5
\end{array}\right]
$$


Using (27), the controller gain $K$ is found to be:

$$
K=\left[\begin{array}{lll}
0.0002 & 0.0084 & -0.0008 \\
0.0003 & 0.0139 & -0.0016
\end{array}\right] \text {. }
$$

Then, solving inequality (21) by using the LMI-Control Toolbox yields:

$$
Q=\left[\begin{array}{ccc}
380.2 & 42.3 & 101.7 \\
42.3 & 413.2 & -40.2 \\
101.7 & -40.2 & 208.2
\end{array}\right]
$$

Using (28), the observer gain $L$ is found to be:

$$
L=\left[\begin{array}{c}
-1.1454 \\
1.4073 \\
-0.5684
\end{array}\right] \text {. }
$$

The performance of system (12) is then simulated using Matlab. The state trajectories are shown in Figs. 1-3. The error trajectories are shown in Figs. 4-6. The trajectories of the controller are shown in Figs. 7- 8. From the figures it can be seen that the state and error

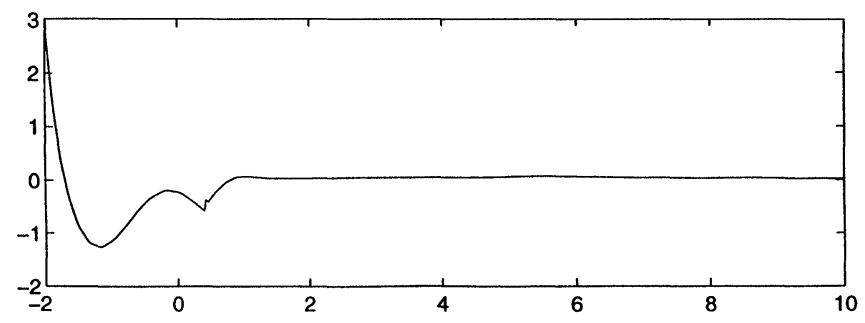

FIGURE 1 Trajectory of state one versus time.

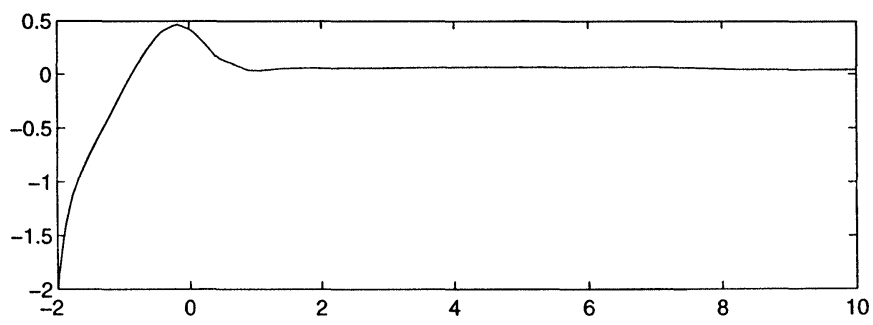

FIGURE 2 Trajectory of state two versus time. 


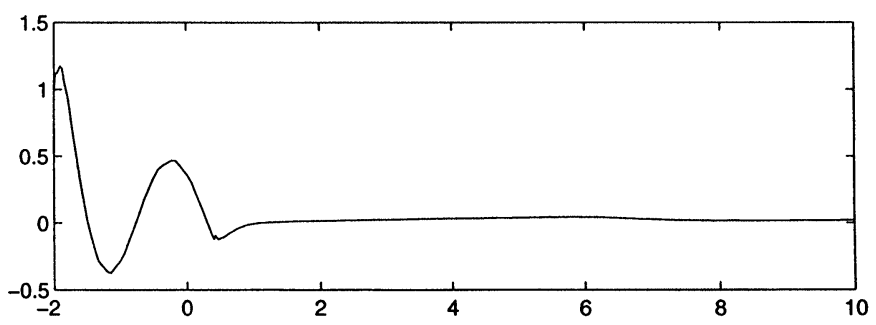

FIGURE 3 Trajectory of state three versus time.

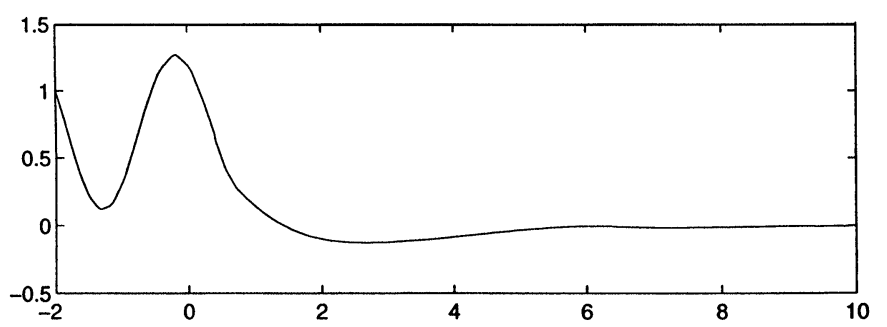

FIGURE 4 Trajectory of error one versus time.

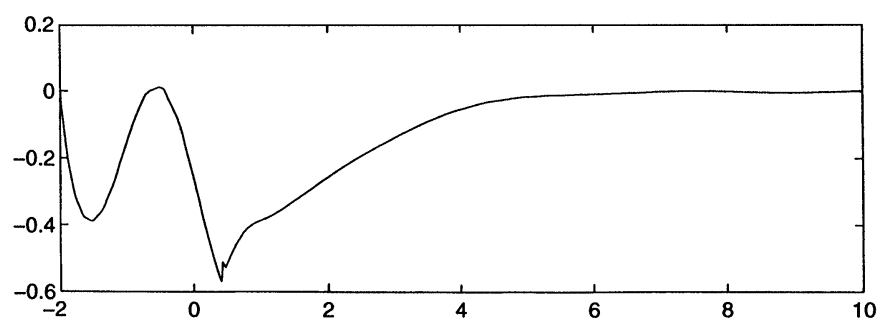

FIGURE 5 Trajectory of error two versus time.

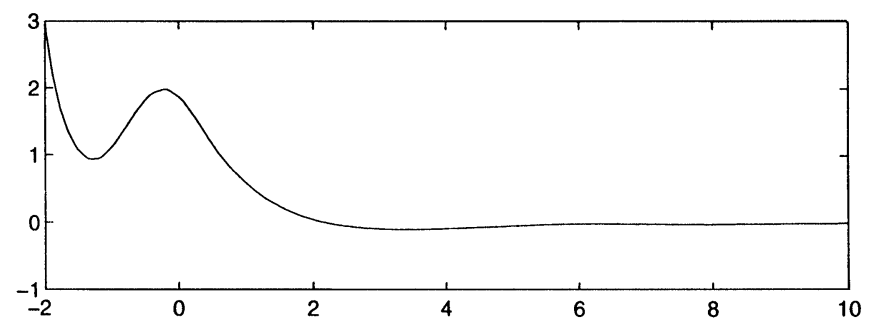

FIGURE 6 Trajectory of error three versus time. 


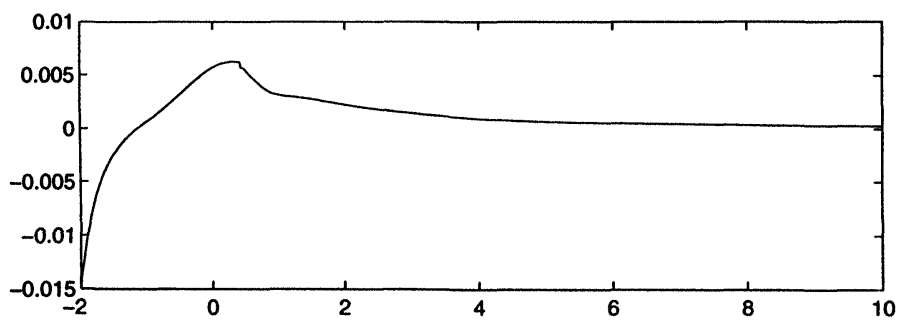

FIGURE 7 Trajectory of input (component 1) versus time.

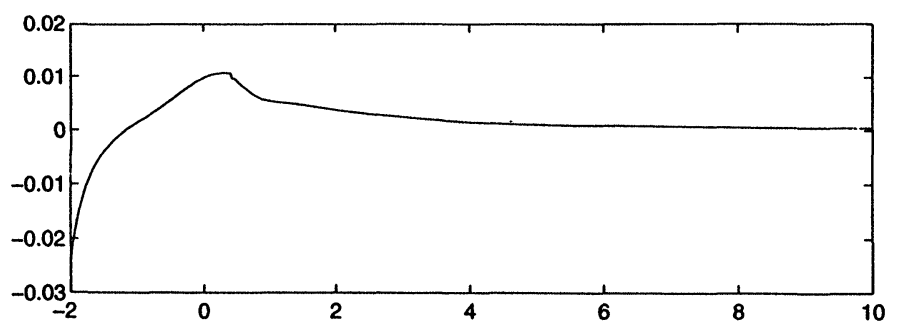

FIGURE 8 Trajectory of input (component 2) versus time.

trajectories converge to zero asymptotically. Hence, it can be concluded that the proposed observer and observer-based controller work quite well for a class of uncertain systems with input and state time lags.

\section{CONCLUSIONS}

This paper has developed a delay-type observer-based controller for a class of uncertain time-lag systems. It is established that the closedloop controlled system is asymptotically stable. The controller and observer gains are determined by solving two LMIs. Simulation results are given to support the theoretical developments.

\section{References}

[1] D.G. Leunberger, “An introduction to observers”, IEEE Trans. Automatic Control, 16(6), 1971, 596-602.

[2] O'Reilly, Observers for Linear Systems, Academic Press, London, 1983.

[3] F. Jabbari and W.E. Schmitendorf, "Effects of using observers on stabilization of uncertain linear systems", IEEE Trans. Automatic Control, 38(2), 1993, 266-271. 
[4] I.R. Petersen, "A Riccati equation approach to the design of stabilizing controllers and observers for a class of uncertain systems", IEEE Trans. Automatic Control, 30, 1985, 904-907.

[5] I.R. Petersen and C.V. Hollot, "High-gain observers applied to problems in stabilization of uncertain linear systems, disturbance attenuation and $H_{\infty}$ optimization", Int. J. of Adaptive Control and Signal Processing, 2, 1988, 347-369.

[6] M.S. Mahmoud, "Output feedback stabilization of uncertain systems with state delay", in Analysis and Synthesis Techniques in Complex Control and Dynamic systems, Vol. 63 of Advances in Theory and Applications, C.T. Leondes (Ed.), 1994, pp. 197-257.

[7] M.S. Mahmoud and M. Zribi, " $H_{\infty}$-controllers for time-delay systems using linear matrix inequalities", J. of Optimization Theory and Applications (JOTA), 1999.

[8] J. Levy-Ramos and A.E. Pearson, "An asymptotic modal observer for linear autonomous time lag systems", IEEE Trans. Automatic Control, 40(7), 1995, $1291-1294$.

[9] H. Trinh and M. Aldeen, "Comments on an asymptotic model observer for linear autonomous time lag systems", IEEE Trans. Automatic Control, 42(5), 1997, $742-745$.

[10] M.S. Mahmoud and M. Zribi, "Stabilizing controllers using observers for uncertain systems with delay", 1999 (submitted).

[11] M.S. Mahmoud, M. Zribi and Y.C. Soh, "Exponential stabilization of state-delay systems with mismatched uncertainties", 1999, IEE Proceedings of Control Theory and Applications.

[12] V. Kolomanovskii and A. Myshkis, Applied Theory of Functional Differential Equations, Kluwer Academic Pub., N.Y., 1986.

[13] N. Gahinet, A. Nemirovski, A.J. Laub and M. Chilali, LMI Control Toolbox for Use with MATLAB, The MathWorks, Inc., 1995. 


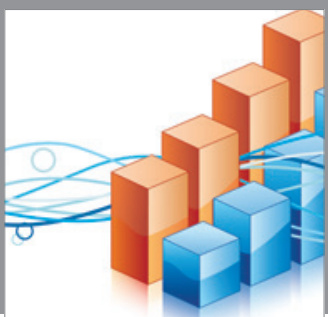

Advances in

Operations Research

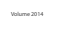

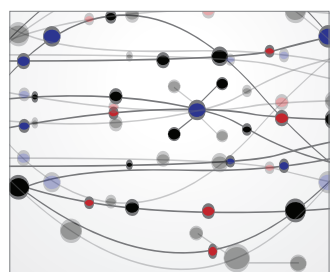

\section{The Scientific} World Journal
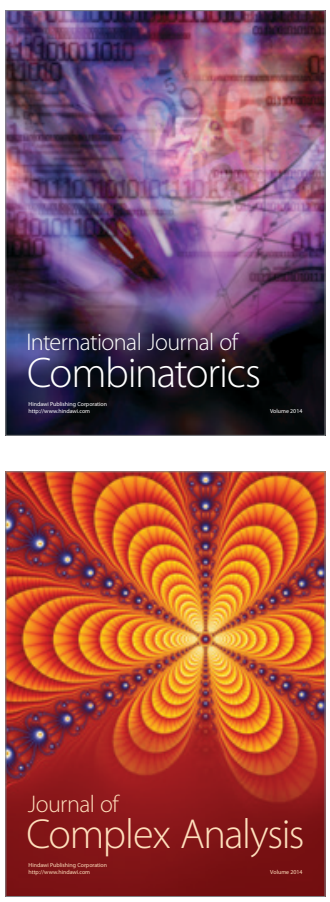

International Journal of

Mathematics and

Mathematical

Sciences
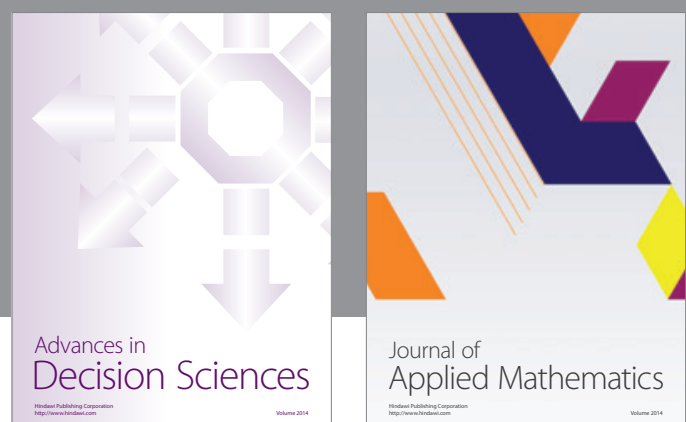

Journal of

Applied Mathematics
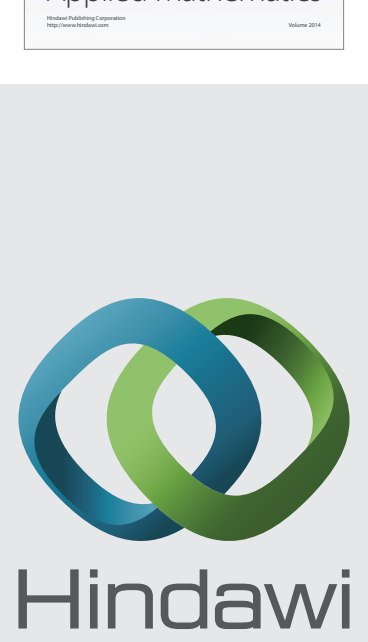

Submit your manuscripts at http://www.hindawi.com
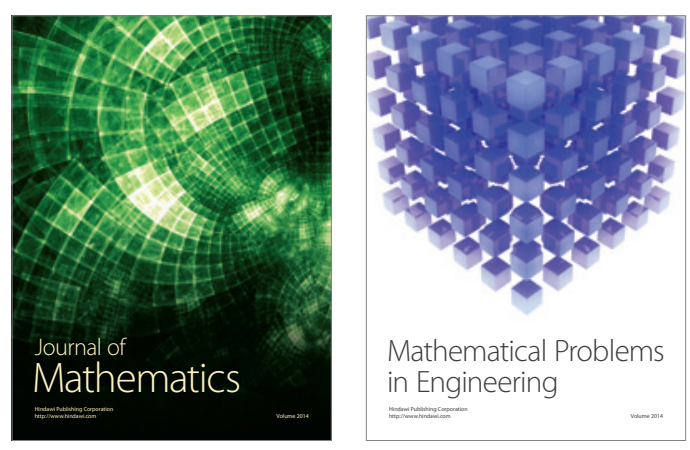

Mathematical Problems in Engineering
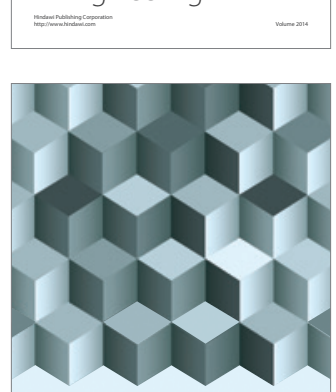

Journal of

Function Spaces
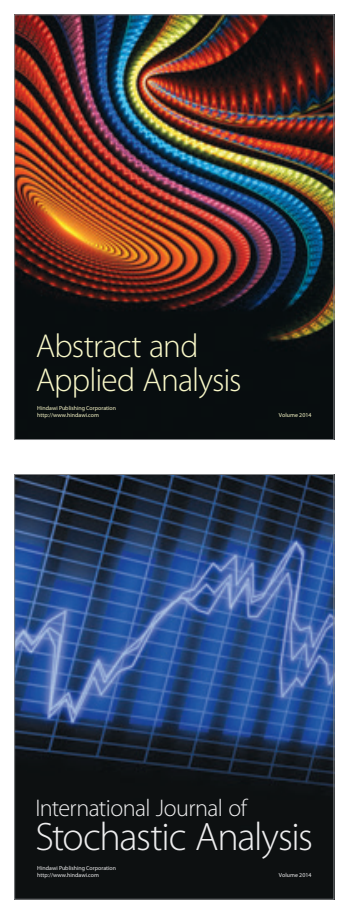

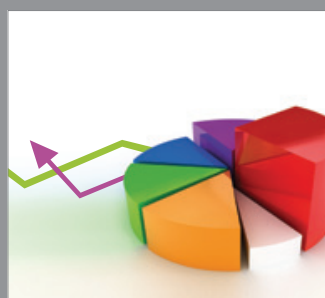

ournal of

Probability and Statistics

Promensencen
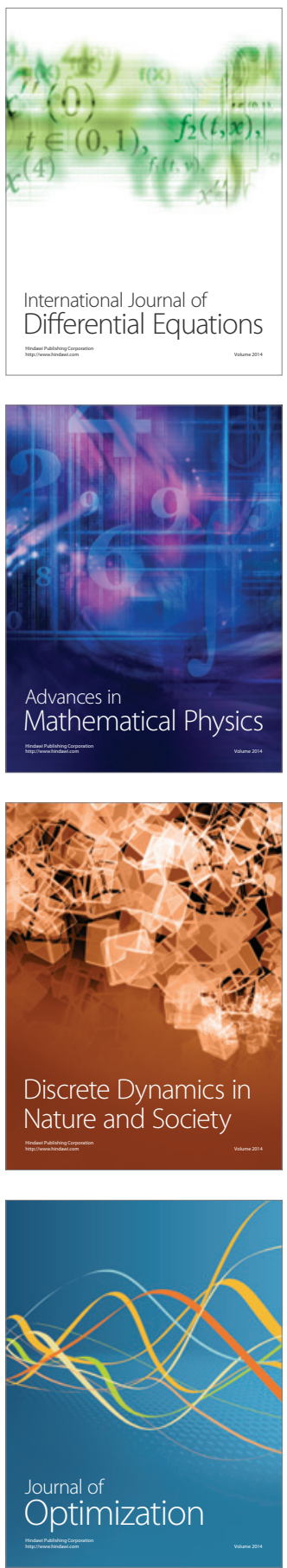